\title{
Methionyl-tRNA Synthetase is a Useful Diagnostic Marker for Lymph Node Metastasis in Non-Small Cell Lung Cancer
}

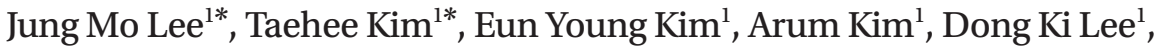 \\ Nam Hoon Kwon' ${ }^{2}$ Sunghoon $\mathrm{Kim}^{2}$, and Yoon Soo Chang ${ }^{1}$ \\ ${ }^{1}$ Department of Internal Medicine, Yonsei University College of Medicine, Seoul; \\ ${ }^{2}$ Medicinal Bioconvergence Research Center, Department of Molecular Medicine and Biopharmaceutical Sciences, \\ Graduate School of Convergence Technology, Seoul National University, Seoul, Korea.
}

Purpose: Identification of lymph node (LN) metastasis in non-small cell lung cancer (NSCLC) is critical for disease staging and selection of therapeutic modalities. Sometimes it is not possible to obtain LN core tissue by endobronchial ultrasound-guided transbronchial needle aspirate (EBUS-TBNA), resulting in low diagnostic yield.

Materials and Methods: In this study, 138 specimens were collected from 108 patients who underwent EBUS-TBNA under the suspicion of LN metastasis of NSCLC. Diagnostic yields of anti-CD45 and anti-methionyl-tRNA synthetase (MRS), immunofluorescent (IF) staining on cytology specimens were compared with those of conventional cytology and positron emission tomography-computed tomography (PET-CT).

Results: MRS was strongly expressed in NSCLC cells metastasized to LNs, but weakly expressed in cells at the periphery of the LN germinal center. The majority of cells were $\mathrm{CD} 20$ positive, although a few cells were either $\mathrm{CD} 3$ or $\mathrm{CD} 14$ positive, indicating that CD45 staining is required for discrimination of non-malignant LN constituent cells from NSCLC cells. When the diagnostic efficacy of MRS/CD45 IF staining was evaluated using $138 \mathrm{LN}$ cellular aspirates from 108 patients through EBUS-TBNA, the sensitivity was $76.7 \%$ and specificity was $90.8 \%$, whereas those of conventional cytology test were $71.8 \%$ and $100.0 \%$, respectively. Combining the results of conventional cytology testing and those of PET-CT showed a sensitivity and specificity of $71.6 \%$ and $100 \%$, and the addition of MRS/CD45 dual IF data to this combination increased sensitivity and specificity to $85.1 \%$ and $97.8 \%$, respectively.

Conclusion: MRS/CD45 dual IF staining showed good diagnostic performance and may be a good tool complementing conventional cytology test for determining LN metastasis of NSCLC.

Key Words: Aminoacyl tRNA synthetase, biomarker, EBUS-TBNA, MRS, NSCLC

Received: July 29, 2019 Revised: September 4, 2019

Accepted: September 11, 2019

Corresponding author: Yoon Soo Chang, MD, PhD, Department of Internal Medicine, Yonsei University College of Medicine, 4th Floor, Research Center for Future Medicine, 63 Gil-20, Eonju-ro, Gangnam-gu, Seoul 06229, Korea.

Tel: 82-2-2019-3309, Fax: 82-2-3463-3882, E-mail: yschang@yuhs.ac

*Jung Mo Lee and Taehee Kim contributed equally to this work.

-The authors have no potential conflicts of interest to disclose.

(C) Copyright: Yonsei University College of Medicine 2019

This is an Open Access article distributed under the terms of the Creative Commons Attribution Non-Commercial License (https://creativecommons.org/licenses/ by-nc/4.0) which permits unrestricted non-commercial use, distribution, and reproduction in any medium, provided the original work is properly cited.

\section{INTRODUCTION}

Lung cancer is the leading cause of cancer death worldwide, with a relative survival rate of about $25 \%$ in the 5 years following the initial diagnosis. ${ }^{1}$ According to American Cancer Society estimates, there will be 222500 new cases of lung cancer in the United States in 2017 and 155870 deaths from lung cancer. ${ }^{2}$ Based on these data, various attempts have been made around the world to develop effective screening methods for the detection of lung cancer still in the treatable stages, with CTbased screening methods currently being widely applied..$^{3-5}$ However, a high false positive rate due to nonspecific lesions 
using these methods requires the urgent development of new and efficient diagnostic methods. ${ }^{6}$

The staging of non-small cell lung cancer (NSCLC) described by the tumor-node-metastasis system is a key process in making prognoses and setting treatment modalities. Recently, endobronchial ultrasound-guided-transbronchial needle aspirate (EBUS-TBNA) assay has been widely applied worldwide, as the detection of cancer cells in the thoracic lymph node (LN) has become a major issue not only in the determination of the cancer stage and in the selection of treatment modalities, but also in the initial diagnosis and selection of drugs at the time of recurrence. $^{?}$

The EBUS-TBNA procedure shows high diagnostic yields when a core biopsy specimen is secured, although diagnosis rates are lowered if only a cytology specimen is secured due to the location of the LN or upon other problems during the procedure. In cases where only cytologic specimens are obtained during the EBUS-TBNA procedure, problems arise in obtaining accurate diagnostic results due to the limited number of neoplastic cells obtained, background interference, etc. Therefore, repeated and consumable procedures are required for a long time in the procedure to obtain sufficient core biopsy specimens. ${ }^{8}$ The rapid on-site evaluation (ROSE) methods that have been developed to overcome some of these problems. However, despite these advantages, ROSE is not widely used because of certain limitations, such as its limited ability to reach confirmatory results, the fact that it evaluates only the usefulness of the sample, and a lack of equipment or manpower. ${ }^{9}$

Aminoacyl-tRNA synthetases (ARSs) are a group of enzymes responsible for protein synthesis and are essential for cell survival and growth. They also have non-canonical functions related to various life phenomena in various type of cells. ${ }^{10}$ Among the ARSs, leucyl-tRNA synthetase, methionyl-tRNA synthetase (MRS), and glutamyl-prolyl tRNA synthetase interact with the proteins in the mTORC1, GCN2, CDK4, and vascular endothelial growth factor receptor (VEGFR) signal pathways, which play critical roles in various cancers. ${ }^{11-13}$ Recently, overexpression of some ARSs have been connected to increased survival in certain cancer patients, ${ }^{14}$ and attempts to treat various cancers using ARS inhibitors have been ongoing. ${ }^{15}$ ARSs are canonically present in the cytoplasm, but are also secreted outside the cell under various signals. The multi-functionality and multi-localization of ARSs makes them useful biomarker candidates in various types of tissue specimens, as well as blood.

MRS, one of the ARSs, is an important enzyme for the initiation of translation and plays an important role in tumor growth. ${ }^{16}$ MRS increases ribosomal RNA biogenesis in the nucleolus and interacts with a variety of signaling molecules, such as mTORC1, GCN2, CDK4, and VEGFR. ${ }^{11,12,17}$ In addition, MRS expression is increased in lung cancer tissues, compared with adjacent, normal-appearing lung tissues, and its overexpression is associated with poor clinical outcomes in lung cancer patients. ${ }^{14}$ Therefore, MRS is considered to be a candi- date for biomarker diagnosis of lung cancer. Therefore, we investigated the usefulness of MRS as a biomarker for diagnosing LN metastasis in NSCLC by evaluating its expression in cytology specimens obtained from the EBUS-TBNA procedure.

\section{MATERIALS AND METHODS}

\section{Antibodies, cells, and other materials}

Anti-MRS antibodies [EPR9873(B)] were obtained from Oncotag (HFTAG201, Suwon, Korea), while the anti-CD3 (2GV6) rabbit monoclonal primary antibody was obtained from Ventana Inc. (790-4341, Arizona, AZ, USA), the Novocastra ${ }^{\mathrm{TM}}$ Liquid Mouse monoclonal antibody CD20 from Leica (NCL-L-CD20L26, Newcastle, UK), the CD14 (5A3B11B5) mouse antibody (sc-58951, Dallas, TX, USA) from Santa Cruz Biotechnology, the human CD45 mouse antibody (MAB1430, Minneapolis, MN, USA) from R\&D systems, and the thyroid transcription factor-1 (SPT24) mouse antibody (PA0364, Newcastle, UK) from Leica Biosystems. The anti-Mouse-AF555 (4409), and anti-rabbit-AF (4412) antibodies were obtained from Cell Signaling Technology (Danvers, MA, USA). Molt-4, Daudi, and H460 cells were obtained from the Korean Cell Line Bank (Seoul, Korea). ThinPrep PreservCyt ${ }^{\mathbb{B}}$ Solution was obtained from Hologic Inc. (\#70097-002, Marlborough, MA, USA) and the Envision Kit and DAB from Dako (\#K3468, Carpinteria, CA, USA).

\section{Study subjects and sample collection}

Study samples were prospectively obtained from patients who 1) visited Severance or Gangnam Severance Hospital, affiliated with Yonsei University, 2) were suspected to have lung cancer, 3) agreed to undergo EBUS-TBNA, 4) had significant LN enlargement (greater than $1.0 \mathrm{~cm}$ in short diameter) on enhanced $\mathrm{CT}$, and 5) provided consent for the use of specimens. After completion of routine cytologic examination and evaluation of the cellular aspirates from EBUS-TBNA by the pathology department, residual cells were mounted on slides using the ThinPrep (Hologic Inc.) procedure and stained. This study was approved by the institutional review board (IRB) of Gangnam Severance Hospital (IRB \#4-2017-0387) and was carried out in compliance with the Declaration of Helsinki and Korean good clinical practice guidelines.

\section{Immunohistochemistry (IHC)}

Sections mounted on the slides were immersed three times for 5 minutes in xylene, two times for 2 minutes in 100\% ethanol, for 2 minutes in $95 \%, 90 \%$, and $70 \%$ ethanol and distilled water, and then for 5 minutes in $1 X$ phosphate-buffered saline (PBS) for deparaffinization and rehydration. After quenching the endogenous peroxidase activity by treatment with $0.3 \%$ hydrogen peroxide for 10 minutes, the antigen was retrieved through immersion in preheated $0.1 \mathrm{M}$ citrate buffer ( $\mathrm{pH}$ 6.0) 
and then boiling for 5 minutes three times at 30-second intervals. The sections were then washed with $1 \mathrm{X}$ PBS-T $(0.3 \%$ Tween-20) and blocked with blocking solution containing $2 \%$ goat serum and $2 \%$ bovine serum albumin in PBS for $30 \mathrm{~min}-$ utes at $4^{\circ} \mathrm{C}$. The primary antibody was diluted with the blocking solution and incubated with the sections at $4^{\circ} \mathrm{C}$ overnight. The dilution factors of the primary antibodies were as follows: MRS, 1:250; CD3 antibody working solution and CD20, 1:100; thyroid transcription factor 1, CD14, and CD45, 1:200. The next day, sections were treated with an Envision+ System-HRP-labelled polymer anti-rabbit/mouse solution (K5007, Dako, Carpinteria, CA, USA) at $4^{\circ} \mathrm{C}$ for 1 hour and visualized with DAB chromogen (Novocastra ${ }^{\mathrm{TM}}$ ). After immersion in Mayer's hematoxylin solution for 1 minute, the sections were washed, dehydrated, and mounted with coverslips.

\section{Immunofluorescence (IF) staining}

ThinPrep slides (Hologic Inc.) were immersed in 1X PBS for 5 minutes and then permeabilized with $0.2 \%$ PBS-T at room temperature for 30 minutes. After washing with $1 X$ PBS, sections were blocked with $2 \%$ goat serum for 1 hour and then incubated with the primary antibody mixture, wherein MRS was diluted at a ratio of 1:250 and CD45 at a ratio of 1:100 in PBS, for $90 \mathrm{~min}$. After washing, sections were incubated with a 1:1000 diluted secondary antibody mixture containing antiRabbit-AF488 and anti-Mouse-AF555 at room temperature for 1 hour and then reacted with 4'6-diamidino-2-phenylindole for 1 minute to counterstain the nucleus. Slides were mounted with coverslips using ProLong Gold Antifade Reagent ${ }^{\circledR}$ (P36930, Invitrogen, Carlsbad, CA, USA) and slides were shaded and stored frozen at $-20^{\circ} \mathrm{C}$. Stained slides were observed using a Carl-Zeiss Imager M2 fluorescence microscope (Imager M2, Carl Zeiss, Oberkochen, Germany) and a Carl-Zeiss LSM 750 confocal microscope (Carl Zeiss), and images were analyzed using ZENlite software (Carl-Zeiss).

\section{Interpretation criteria and statistical analysis}

The following four criteria were applied for the classification of cells as NSCLC: 1 ) a nucleus diameter $\geq 10 \mu \mathrm{m}, 2$ ) no indentation in the nucleus, 3) prominent nucleoli, and 4) MRS positivity and CD45 negativity. The MRS and CD45 staining results of clinical specimens were interpreted by comparing the staining intensity of the reference sample, which comprised a 1:1:1 mixture of H460 NSCLC cells, Molt-4 T-cell lymphoblastic cells, and Daudi B lymphoblastic cells. MRS was expressed in all cells in the reference sample, and clinical specimens were deemed positive when stained with the same or higher intensity than the reference. CD45 was expressed in two thirds of the cells in the reference sample, except H460 cells, and clinical specimens were deemed positive when staining intensity was the same or higher than that of the reference sample. Two independent researchers (A Kim and JM Lee) who were blinded to the pathological reports read the slides, and in case of discrepancy between the results of these two investigators, a third researcher (YS Chang) also evaluated the slides. The sensitivity and specificity of MRS/CD45 IF staining, conventional cytology test, and PET-CT were compared with the those of EBUS-TBNA core biopsies, a gold standard.

\section{RESULTS}

\section{MRS strongly expressed in NSCLC cells metastasized to the LNs}

Previous studies have shown that MRS is overexpressed in about $77.4 \%$ of NSCLC tissues. ${ }^{14}$ To investigate whether these findings were also observable in NSCLC cells metastasized to the LNs, MRS IHC staining was performed in five LN surgical specimens that were confirmed to harbor NSCLC cell metastasis. In all five samples, MRS was clearly overexpressed in the cancer cell cytoplasm, compared to non-malignant cells comprising LN structures (Fig. 1). Meanwhile, however, weak MRS expression was detected in cells other than NSCLC cells in the LNs.

\section{CD45 as a useful biomarker for differentiating other lymphoid structures expressing NSCLC and MRS}

In order to develop a method for distinguishing MRS-expressing LN constructs from NSCLC cells in LNs, additional IHC staining was performed using biomarkers for the cells constituting the lymphoid system (Fig. 2). Because the periphery of germinal centers that showed weak MRS expression was matched with the region where mature B-cells are primarily found, IHC staining for CD20, a B-lymphocyte marker, was carried out. Interestingly, areas that were weakly positive for MRS well corresponded with CD20-positive areas. To clarify whether CD20-positive B lymphocytes were the only cells expressing MRS, further IHC studies were performed using thymic tissues, where T-cells are abundant, obtained during the operation of thymoma and B-cell enriched splenic tissues. In these tissues, the expression of MRS corresponded with that of CD20; however, a few cells weakly expressing MRS were also observed in the deep paracortical area and trabeculae where T-lymphocytes and macrophages/monocytes are present. Additional IHC staining for MRS, CD3, CD14, and CD20 was performed in tonsil tissue, which is structurally similar to lymphoid systems in the LN. Similar to the results seen in the LN, splenic, and thymic tissues, MRS expression and CD20 expression were most often co-localized, although co-localization with MRS was also more rarely observed in CD3- and CD14-expressing cells. This suggests that CD20 expression alone could not differentiate LN cells expressing MRS nor NSCLC cells. Therefore, IHC staining was performed using the leukocyte common antigen marker CD45, and the results showed that MRS expression in areas other than in NSCLC cells was well co-localized with CD45-positive areas. 


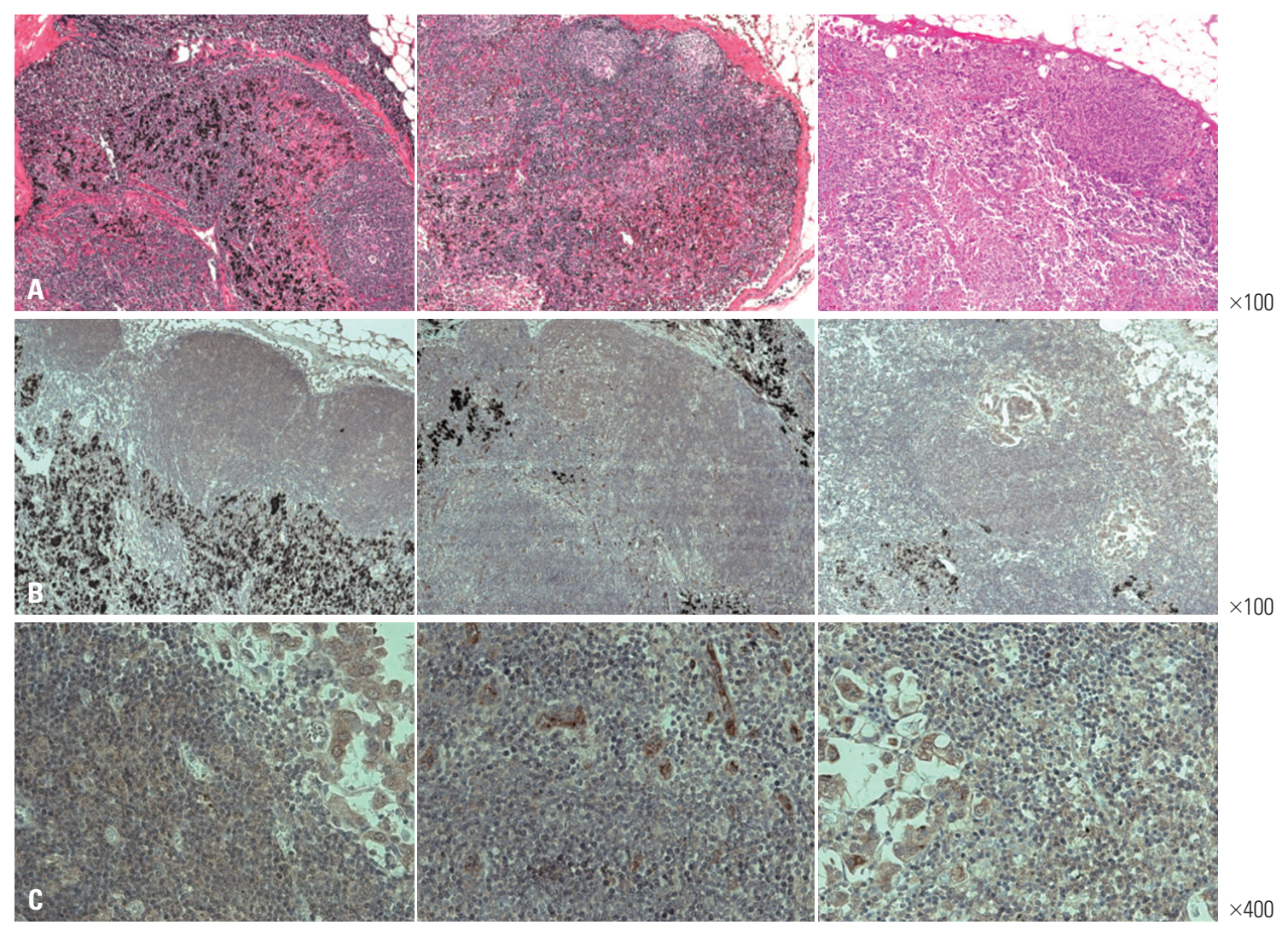

Fig. 1. Hematoxylin and eosin stain (A) and methionyl-tRNA synthetase immunohistochemistry $(B$ and $C)$ staining of three lymph node specimens of non-small cell lung cancer metastasis. Each column represents the same case $(A$ and $B: \times 100, C: \times 400)$. Representative cases are shown.

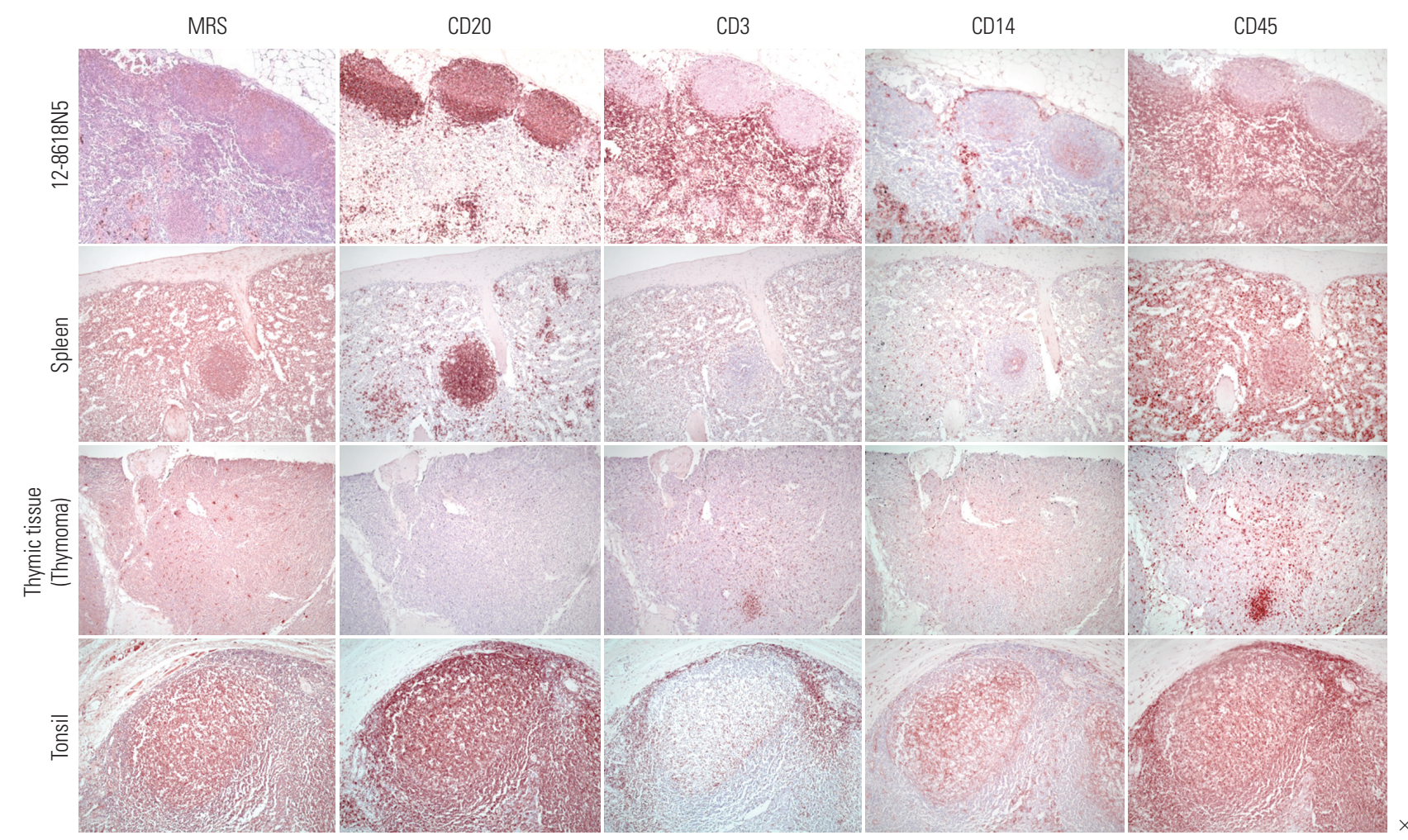

Fig. 2. Immunohistochemistry for methionyl-tRNA synthetase (MRS), CD20 (B-lymphocytes), CD3 (T-lymphocytes), CD14 (macrophages and monocytes), and CD45 (leukocyte common antigen) markers in the lymph nodes with non-small cell lung cancer, splenic tissue, thymic tissue from the thymoma, and tonsils. Magnification: $\times 100$. 


\section{Preparation of reference specimens using MRS- and CD45-expressing cells}

We hypothesized that MRS and CD45 double staining would be appropriate for confirming the presence of $\mathrm{LN}$ metastasis of NSCLC. MRS and CD45 double stained cells are leukocyte lineage cells, and MRS positive and CD45 negative cells were judged to be NSCLC cells, which was established as one of the major criteria for judging NSCLC cells, in addition to nucleus size, shape, and nucleoli characteristics, as described in the materials and methods section. To apply these findings to the interpretation of cytologic samples obtained from EBUS-TBNA, a 1:1:1 mixture of H460, Daudi, and Molt-4 cells was processed as described previously, and ThinPrep ${ }^{\circledR}$ slides (Hologic Inc.) of this mixture were prepared and used as a reference sample for IHC and IF staining of clinical specimens (Fig. 3A). When the reference samples were stained for MRS and CD45, the ratio of MRS positive to MRS/CD45 double positive cells was $1: 2$. When studying the clinical specimens, reference samples were stained simultaneously and used for qualitative interpretation of cell staining in the cytological specimens (Fig. $3 \mathrm{~B}$ and $\mathrm{C})$.

\section{Application of MRS/CD45 dual IF staining to clinical specimens}

EBUS-TBNA was performed for 138 LNs from 108 patients (Table 1). Among the 138 LNs biopsied, lung cancer metastasis was confirmed in 74 cases (53.6\%), and no evidence of cancer was found in 64 cases (46.4\%). Among the cases with cancer metastasis, adenocarcinoma was the most common (71.6\%), followed by squamous cell carcinoma (25.7\%) and others (2.7\%). The locations of the LNs where EBUS-TBNA was performed were the subcarinal LNs in 54 (39.1\%) cases, paratracheal LNs in $52(37.7 \%)$ cases, and hilar or interlobar LNs in $32(23.2 \%)$ cases. Representative IF images of MRS and CD45 from EBUSTBNA cytologic specimens are shown in Fig. 3D and E. Using EBUS-TBNA core tissue biopsy results as a reference, the results of MRS/CD45 IF staining were compared with those of conventional cytology test and PET-CT imaging. The sensitivi-

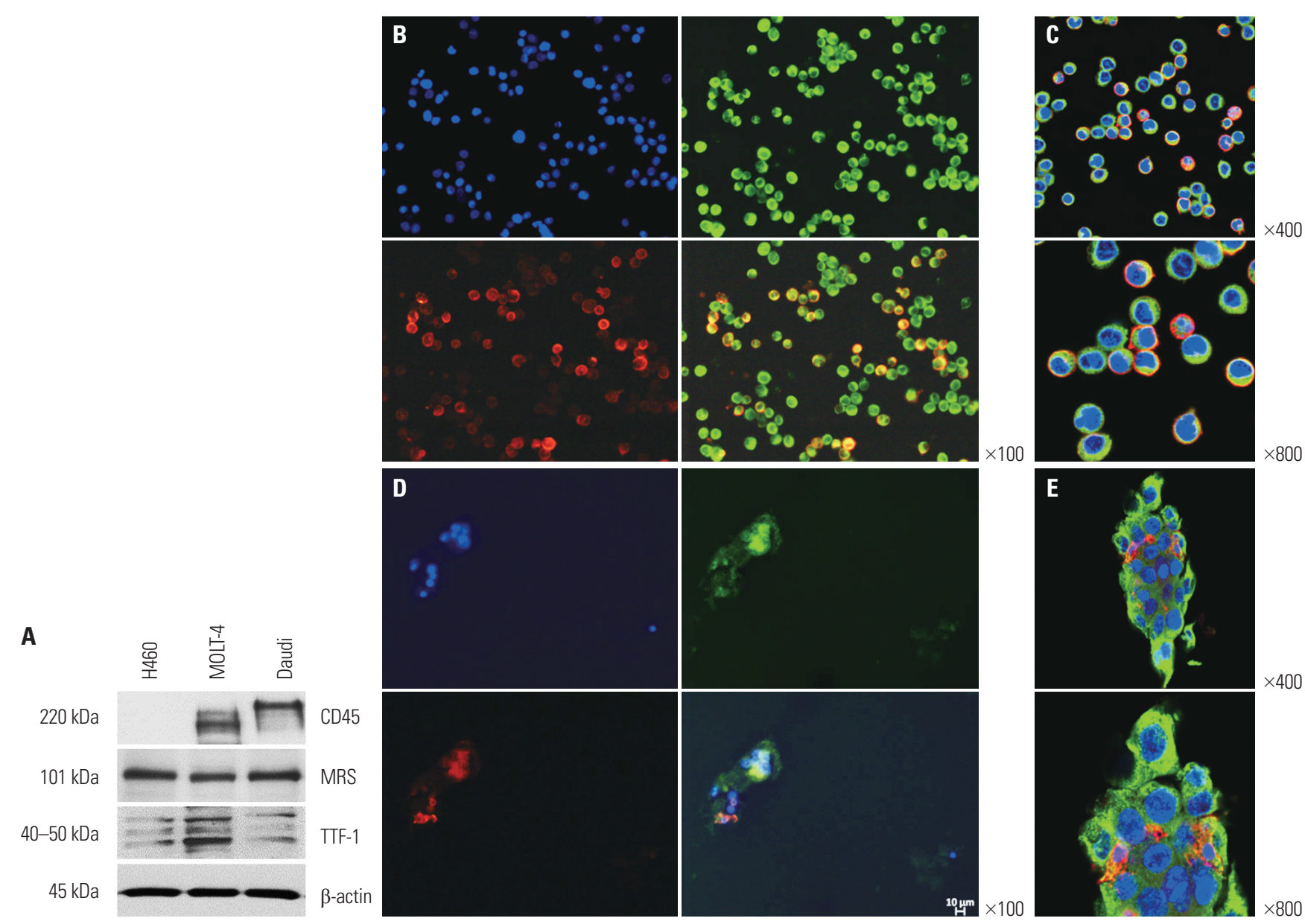

Fig. 3. Immunoblotting of methionyl-tRNA synthetase (MRS)-positive H460 cells, CD45/CD3-positive Molt-4 cells, and CD45/CD20-positive Daudi cells (A). Fluorescent immunochemical staining for MRS and CD45 in the reference samples containing a fixed ratio of H460, Daudi, and Molt-4 cells (B and C) and endobronchial ultrasound-guided transbronchial needle aspirate-derived samples ( $D$ and E). Images were taken using a fluorescent microscope (B and D) and confocal microscope ( $C$ and $E$ ). Blue represents nucleus (4', 6-Diamidine-2'-phenylindole dihydrochloride: DAPI); green, MRS; and red, CD45. B and D confocal images show merged images only. TTF-1, thyroid transcription factor 1. 
ty of MRS/CD45 IF staining was $76.7 \%$, and the specificity was $90.8 \%$. In contrast, those for conventional cytology were $71.8 \%$ and $100.0 \%$, respectively. PET-CT imaging showed a sensitivity and specificity of $88.4 \%$ and $63.0 \%$, respectively. Combining the results of MRS/CD45 IF staining with that of conventional cytology test increased sensitivity and specificity to $87.3 \%$ and $91.9 \%$, respectively. When combining the results of cytology and PET-CT, which is commonly applied in medical practice, the sensitivity and specificity were $71.6 \%$ and $100 \%$, respectively, and the addition of MRS/CD45 IF to these results resulted in a sensitivity and specificity of $85.1 \%$ and $97.8 \%$, respectively (Tables 2 and 3, Fig. 4). Taken together, while MRS/CD45 IF staining was more sensitive than conventional cytology, the specificity was lower; meanwhile, in comparison with PET-CT imaging, the sensitivity of MRS/CD45 IF staining was lower, but its specificity was higher.

\section{DISCUSSION}

Accurate mediastinal LN staging is important for disease staging and the selection of appropriate treatment modalities in lung cancer patients. ${ }^{18}$ In the EBUS-TBNA procedure, the diagnostic yield increases when a core tissue specimen is secured, whereas when only cytology specimens are obtained, the diagnosis rate decreases. Approximately 15 to $20 \%$ of $\mathrm{LN}$ aspi-

Table 1. Demographic Characteristics of the Study Cases

\begin{tabular}{lc}
\hline \multicolumn{1}{c}{ Baseline characteristics } & Values \\
\hline No. of patients & 108 \\
Age (yr) (range) & $69.0(61.0-6.0)$ \\
Men (n, \%) & $68(63.0)$ \\
Serum CEA level (ng/mL) (range) & $3.5(1.9-10.6)$ \\
No. of LNs & 138 \\
Location of LNs (n, \%) & \\
\hline Paratracheal LN & $52(37.7)$ \\
Subcarinal LN & $54(39.1)$ \\
$\quad$ Hilar, interlobar LN & $32(23.2)$ \\
Core pathology (n, \%) & \\
\hline No evidence of cancer & $64(46.4)$ \\
Adenocarcinoma & $53(38.4)$ \\
Squamous cell carcinoma & $19(13.8)$ \\
\hline Poorly differentiated carcinoma & $2(1.4)$ \\
\hline
\end{tabular}

CEA, carcinoembryonic antigen; LN, lymph node.

Values are presented as median (range) or $\mathrm{n}(\%)$ unless otherwise indicated. rates result in non-diagnostic quality specimens. ${ }^{19,20}$ In addition, a lot of time and labor are required to obtain a core tissue biopsy specimen, and the rate of complications increases accordingly. Since EBUS-TBNA is an invasive and costly test, it is necessary to develop an accurate diagnostic method using cytologic specimens. Here, surplus cytologic specimens were prospectively collected from patients who underwent EBUS-TBNA under the suspicion of LN metastasis of lung cancer and were used to explore the possibility of MRS as a biomarker of LN metastasis in lung cancer.

ARSs are housekeeping enzymes that catalyze the ligation of amino acids to their cognate tRNAs with high fidelity. ${ }^{11}$ Based on the roles of ARSs, their overexpression is expected to affect the growth and progression of cancer, which has an increased metabolic activity. Among them, MRS is a major enzyme in the initiation of ARS protein synthesis, transferring a Met to the initiator tRNA (tRNA ${ }_{i}^{\mathrm{Met}}$ ). Dysfunctions of MRS have been observed in interstitial lung disease, Charcot-Marie-Tooth disease, and pulmonary alveolar proteinosis. ${ }^{21,22}$

Previously, our group observed overexpression of MRS in mouse lung cancer models and human tissue obtained through surgery, and confirmed that this overexpression was associated with a poor prognosis in NSCLC patients. ${ }^{14}$ In this study, we confirmed the possibility of MRS as a biomarker for lung cancer by confirming its expression in aspirated cell specimens, which can be secured safely from EBUS-TBNA. To date, the relationship between MRS and LN metastasis is, as of yet, unknown, although we suspect that it may affect cancer progression by increasing cell turnover. Based on the expression patterns of MRS in these needle aspirate cytology specimens, its usage as a biomarker might be able to be extended to other cytology specimens, such as pleural effusion and bronchoalveolar lavage specimens, to aid in the diagnosis of lung cancer.

MRS overexpression was mainly observed in lung cancer

Table 3. Comparison of Diagnostic Yields

\begin{tabular}{lccc}
\hline & $\begin{array}{c}\text { Sensitivity } \\
(\%)\end{array}$ & $\begin{array}{c}\text { Specificity } \\
(\%)\end{array}$ & AUC \\
\hline MRS/CD45 IF staining & 76.7 & 90.8 & 0.837 \\
Cytology & 71.8 & 100.0 & 0.859 \\
PET-CT & 88.4 & 63.0 & 0.747 \\
Cytology+PET-CT & 71.6 & 100.0 & 0.925 \\
Cytology+PET+MRS/CD45 IF staining & 85.1 & 97.8 & 0.952 \\
\hline
\end{tabular}

AUC, area under the curve; MRS, methionyl-tRNA synthetase; IF, immunofluorescent; PET-CT, positron emission tomography-computed tomography.

Table 2. Comparison of Results

\begin{tabular}{|c|c|c|c|c|c|c|}
\hline \multirow{2}{*}{ Core pathologic results } & \multicolumn{2}{|c|}{ MRS IF staining } & \multicolumn{2}{|c|}{ Conventional cytology } & \multicolumn{2}{|c|}{ PET-CT ${ }^{\dagger}$} \\
\hline & Positive & Negative & Positive & Negative & Positive & Negative \\
\hline Malignant lesion & 56 & 17 & 51 & 20 & 61 & 8 \\
\hline Benign lesion & 6 & 59 & 0 & 62 & 17 & 29 \\
\hline
\end{tabular}

MRS, methionyl-tRNA synthetase; IF, immunofluorescent; PET-CT, positron emission tomography-computed tomography.

${ }^{*}$ Cytologic results for five lesions were not available; ${ }^{+P E T-C T ~ o f ~} 23$ lesions was not available. 

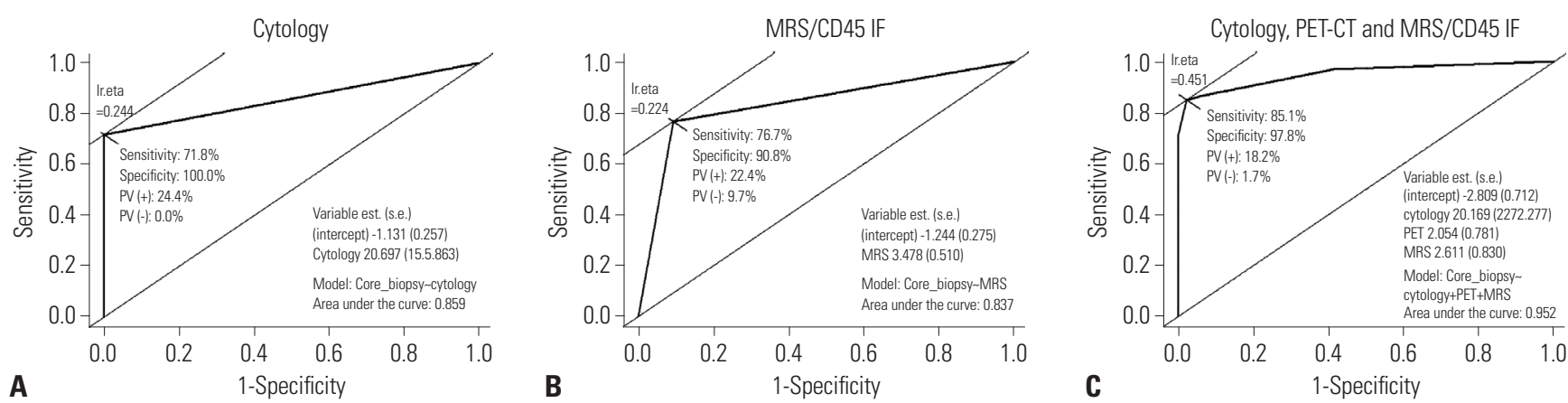

Fig. 4. Receiver operating characteristic (ROC) curves of predictors of lung cancer lymph node metastasis. ROC curves of (A) cytology, (B) methionyltRNA synthetase (MRS)/CD45 immunofluorescence (IF), and (C) the combination of cytology, positron emission tomography (PET), and MRS/CD45 IF were created using core biopsy results as a reference value. Ir.eta, linear predictor eta; $P V(+)$, positive predictive value; PV(-), negative predictive value; s.e., standard error; est., estimates.

cells, although weak expression was observed in other cells comprising the LN, suggesting that there is a limit to the use of MRS alone as a diagnostic biomarker. Additional markers were developed to complement this, and we confirmed that double staining with CD45 can complement false positive results of MRS in non-cancer cells. Using various lymphoid tissues, such as those from the spleen, thymoma, and tonsils, we found that the majority of cells that express MRS were CD20-positive Bcells. Considering the role of CD20-positive B-cell synthesizing immunoglobulins, MRS positivity in that subset of lymphoid cells is predictable. T-cells and other immune system cells also showed intermittent expression of MRS, which would be expected in a metabolically active state.

MRS and CD45 double IF staining showed specificity for lung cancer cells on thin-prep specimens from EBUS-TBNA procedures, and the results showed higher sensitivity than those of conventional cytological examination, suggesting that MRS/ CD45 staining could be a good candidate as a complementary diagnostic tool for the detection of LN metastasis in lung cancer. Occasionally, the EBUS-TBNA method does not procure the appropriate core tissue specimen and acquires only the cell specimen. In this case, this method could be applied as an assistant method for diagnosis. In medical practice, it is not uncommon for cases in which an initial routine examination reports a non-malignancy that is confirmed as lung cancer later. For example, in this study, a case with a negative result of EBUS-TBNA cytology test and core biopsy showed mild to moderately increased uptake at right lower paratracheal LN in PET-CT. However, this patient had positive MRS/CD45 IF staining, and the final histopathologic diagnosis was squamous cell carcinoma (Supplementary Fig. 1, only online). In addition, this method could be applied as a basis for the development of ROSE to determine the suitability of EBUS-derived cytological specimens. ${ }^{23}$

Considering that these results were obtained from residual specimens that were used and left after for routine cytopathology studies, better diagnostic yield might be expected if appropriate samples are used in the future. Along with CD45/ MRS dual staining positivity, a nuclear diameter of more than
$10 \mu \mathrm{m}$ and the presence of nucleoli were included in our diagnostic criteria. This suggests that a small proportion of small cell lung cancer cells, the nuclear diameter of which is $9.2 \pm 2.1$ $\mu \mathrm{m}$, might be of use, along with CD45/MRS dual staining. ${ }^{24}$ In addition, CD45-negativity has been observed in Reed-Sternberg cells and their variants, as well as some anaplastic large cell lymphomas. ${ }^{25}$ This indicates the need for careful inspection of the nucleus, including the presence of nucleoli, as well as complementary studies, such as radiologic findings and clinical manifestations, in cases where a homogenous population of MRS-positive and CD45-negative cells is observed.

In order to detect metastasis of NSCLC to LNs, a pathologist's experience is critical. To improve diagnostic yield when only a small number of cells is available, MRS/CD45 dual IF staining may be a good complementary test.

In conclusion, double IF staining for MRS and CD45 showed promising diagnostic performance and may be of use as a complementary test to conventional cytological tests in order to determine metastasis of lung cancer to the LNs when only limited cytologic specimen is available.

\section{ACKNOWLEDGEMENTS}

This study was supported by a National Research Foundation of Korea (NRF) grant funded by the Korea government (MSIT) (No. NRF-2014M3A6A4074817, NRF-M3A6A4-2010-0029785, NRF-2015M3A6A4065724, and NRF-2017M3A9F7079378).

\section{AUTHOR CONTRIBUTIONS}

Conceptualization: Nam Hoon Kwon and Sunghoon Kim. Data curation: Jung Mo Lee and Eun Young Kim. Formal analysis: Jung Mo Lee. Funding acquisition: Sunghoon Kim. Investigation: Jung Mo Lee and Taehee Kim. Methodology: Arum Kim. Project administration: Nam Hoon Kwon and Dong Ki Lee. Resources: Yoon Soo Chang. Supervision: Dong Ki Lee. Validation: Yoon Soo Chang. Visualization: Jung Mo Lee and Taehee Kim. Writing_original draft: Jung Mo Lee. Writing_review \& editing: Taehee Kim and Yoon Soo Chang. 


\section{ORCID iDs}

\begin{abstract}
Jung Mo Lee Taehee Kim Eun Young Kim Arum Kim Dong Ki Lee Nam Hoon Kwon Sunghoon Kim

Yoon Soo Chang

https://orcid.org/0000-0002-9972-8506 https://orcid.org/0000-0002-3673-7595 https://orcid.org/0000-0002-3281-5744 https://orcid.org/0000-0002-6220-4717 https://orcid.org/0000-0002-0048-9112 https://orcid.org/0000-0001-6360-2584 https://orcid.org/0000-0002-1570-3230 https://orcid.org/0000-0003-3340-4223
\end{abstract}

\section{REFERENCES}

1. Torre LA, Bray F, Siegel RL, Ferlay J, Lortet-Tieulent J, Jemal A. Global cancer statistics, 2012. CA Cancer J Clin 2015;65:87-108.

2. Siegel RL, Miller KD, Jemal A. Cancer statistics, 2017. CA Cancer J Clin 2017;67:7-30.

3. National Lung Screening Trial Research Team, Aberle DR, Adams AM, Berg CD, Black WC, Clapp JD, et al. Reduced lung-cancer mortality with low-dose computed tomographic screening. N Engl J Med 2011;365:395-409.

4. Horeweg N, van Rosmalen J, Heuvelmans MA, van der Aalst CM, Vliegenthart R, Scholten ET, et al. Lung cancer probability in patients with CT-detected pulmonary nodules: a prespecified analysis of data from the NELSON trial of low-dose CT screening. Lancet Oncol 2014;15:1332-41.

5. Infante M, Cavuto S, Lutman FR, Passera E, Chiarenza M, Chiesa $\mathrm{G}$, et al. Long-term follow-up results of the DANTE trial, a randomized study of lung cancer screening with spiral computed tomography. Am J Respir Crit Care Med 2015;191:1166-75.

6. Aberle DR, Abtin F, Brown K. Computed tomography screening for lung cancer: has it finally arrived? Implications of the national lung screening trial. J Clin Oncol 2013;31:1002-8.

7. Evison M, Morris J, Martin J, Shah R, Barber PV, Booton R, et al. Nodal staging in lung cancer: a risk stratification model for lymph nodes classified as negative by EBUS-TBNA. J Thorac Oncol 2015; 10:126-33.

8. Trisolini R, Cancellieri A, Tinelli C, de Biase D, Valentini I, Casadei $\mathrm{G}$, et al. Randomized trial of endobronchial ultrasound-guided transbronchial needle aspiration with and without rapid on-site evaluation for lung cancer genotyping. Chest 2015;148:1430-7.

9. Bonifazi M, Sediari M, Ferretti M, Poidomani G, Tramacere I, Mei F, et al. The role of the pulmonologist in rapid on-site cytologic evaluation of transbronchial needle aspiration: a prospective study. Chest 2014;145:60-5.

10. Kim S, You S, Hwang D. Aminoacyl-tRNA synthetases and tumorigenesis: more than housekeeping. Nat Rev Cancer 2011;11:708-18.

11. Han JM, Jeong SJ, Park MC, Kim G, Kwon NH, Kim HK, et al. Leucyl-tRNA synthetase is an intracellular leucine sensor for the mTORC1-signaling pathway. Cell 2012;149:410-24.
12. Yannay-Cohen N, Carmi-Levy I, Kay G, Yang CM, Han JM, Kemeny $\mathrm{DM}$, et al. LysRS serves as a key signaling molecule in the immune response by regulating gene expression. Mol Cell 2009;34:603-11.

13. Keller TL, Zocco D, Sundrud MS, Hendrick M, Edenius M, Yum J, et al. Halofuginone and other febrifugine derivatives inhibit prolyl-tRNA synthetase. Nat Chem Biol 2012;8:311-7.

14. Kim EY, Jung JY, Kim A, Kim K, Chang YS. Methionyl-tRNA synthetase overexpression is associated with poor clinical outcomes in non-small cell lung cancer. BMC Cancer 2017;17:467.

15. Kim JH, Lee C, Lee M, Wang H, Kim K, Park SJ, et al. Control of leucine-dependent mTORCl pathway through chemical intervention of leucyl-tRNA synthetase and RagD interaction. Nat Commun 2017;8:732.

16. Kwon NH, Kang T, Lee JY, Kim HH, Kim HR, Hong J, et al. Dual role of methionyl-tRNA synthetase in the regulation of translation and tumor suppressor activity of aminoacyl-tRNA synthetase-interacting multifunctional protein-3. Proc Natl Acad Sci U S A 2011;108: 19635-40.

17. Dehm SM. mRNA splicing variants: exploiting modularity to outwit cancer therapy. Cancer Res 2013;73:5309-14.

18. Um SW, Kim HK, Jung SH, Han J, Lee KJ, Park HY, et al. Endobronchial ultrasound versus mediastinoscopy for mediastinal nodal staging of non-small-cell lung cancer. J Thorac Oncol 2015;10:331-7.

19. Nayak A, Sugrue C, Koenig S, Wasserman PG, Hoda S, Morgenstern NJ. Endobronchial ultrasound-guided transbronchial needle aspirate (EBUS-TBNA): a proposal for on-site adequacy criteria. Diagn Cytopathol 2012;40:128-37.

20. Alsharif M, Andrade RS, Groth SS, Stelow EB, Pambuccian SE. Endobronchial ultrasound-guided transbronchial fine-needle aspiration: the University of Minnesota experience, with emphasis on usefulness, adequacy assessment, and diagnostic difficulties. Am J Clin Pathol 2008;130:434-43.

21. Hadchouel A, Wieland T, Griese M, Baruffini E, Lorenz-Depiereux B, Enaud L, et al. Biallelic mutations of methionyl-tRNA synthetase cause a specific type of pulmonary alveolar proteinosis prevalent on Réunion Island. Am J Hum Genet 2015;96:826-31.

22. Sun Y, Hu G, Luo J, Fang D, Yu Y, Wang X, et al. Mutations in methionyl-tRNA synthetase gene in a Chinese family with interstitial lung and liver disease, postnatal growth failure and anemia. J Hum Genet 2017;62:647-51.

23. Mallya V, Kumar SP, Meganathan P, Shivkumar S, Mehta R. The utility of ROSE (rapid on-site evaluation) in endobronchial ultrasound (EBUS)-guided transbronchial needle aspiration (TBNA): is the picture rosy? J Cytol 2015;32:230-3.

24. Lee TK, Esinhart JD, Blackburn LD, Silverman JF. The size of small cell lung carcinoma cells. Ratio to lymphocytes and correlation with specimen size and crush artifact. Anal Quant Cytol Histol 1992;14: 32-4.

25. Schmid C, Pan L, Diss T, Isaacson PG. Expression of B-cell antigens by Hodgkin's and Reed-Sternberg cells. Am J Pathol 1991;139: 701-7. 